\title{
DISEÑO DE UNA APLICACIÓN INFORMÁTICA PARA PROCESAR LOS REGISTROS OBTENIDOS POR EL DISDRÓMETRO ÓPTICO THIES LPM
}

\author{
Fernández-Raga, María ${ }^{(1)}$, Alfonso Cendón, Javier ${ }^{(2)}$, Fraile Laiz, Roberto ${ }^{(3)}$.
}

(1) Departamento de Química y Física Aplicadas, Universidad de León, mferr@unileon.es

(2) Departamento Ing. Mecánica, Informática y Aeroespacial, Universidad de León, Javier.alfonso@unileon.es

${ }^{(3)}$ Departamento de Química y Física Aplicadas, Universidad de León, rfral@unileon.es

\section{Resumen}

La obtención y gestión de bases de datos completas que recojan datos de las características de las gotas de los diferentes eventos de lluvia en cada lugar concreto resulta sumamente útil en Meteorología.

La recogida de estos datos exige de instrumentos meteorológicos especializados, como los disdrómetros, y concretamente el disdrómetro óptico Laser Precipitation Monitor (LPM) de Thies Clima es uno de los más comunes. Este disdrómetro ha sido utilizado por la Universidad de León para el estudio de las gotas desde el año 2006, generando una base de datos tan grande y pesada que resulta muy complicada de gestionar y analizar.

Es por tanto de alto interés para fines de investigación diseñar un programa informático que gestione las bases de datos y que permita realizar consultas de periodos de interés de una manera automática, extrayendo aquellas estadísticas y valores que resulten de mayor utilidad.

Por lo tanto, el objetivo de este trabajo es diseñar una aplicación que permita procesar los registros obtenidos durante más de 10 años por un disdrómetro LPM Thies clima, y almacenarlos en texto plano en datos válidos para su posterior tratamiento. Para ello, hacemos uso del lenguaje Java que lee los datos, los procesa y los sube al servidor de base de datos. Una vez estos datos son almacenados, se pasan a mostrarse en una aplicación Web que, de forma gráfica, puede presentarlos por fechas, tamaños o velocidades, aportando una información válida para estudio de las precipitaciones. Esta aplicación fue implementada con un Login de seguridad para controlar el acceso de usuarios, y con un interfaz de usuario sencillo que permite que usuarios sin conocimientos en Java puedan ejecutar la aplicación sin ningún tipo de problema.

Palabras clave: disdrómetro, software, gestión de datos, tamaño de gotas, velocidad de gotas.

\section{Introducción}

El estudio de la caracterización de la lluvia requiere la medición de parámetros como son el diámetro de cada gota, la velocidad y el número total de gotas en cada minuto. Para la medición de estos parámetros usamos los disdrómetros, y concretamente el Thies "Laser Precipitation Monitor" (Chen et al, 2015, Frasson et al, 2014). Con este aparato es posible analizar los eventos de precipitación y comparar los resultados obtenidos en diferentes fechas. Pero estos instrumentos arrojan una gran cantidad de datos 
a cada minuto, ya que clasifican cada gota detectada en veintidós tamaños diferentes y veinte velocidades distintas, generando una enorme cantidad de datos, lo que supone una gran dificultad en el manejo y análisis de un volumen de datos tan grande. Además, se da la circunstancia de que este problema se multiplica al disponer de datos midiendo en distintas ciudades, como es el caso en numerosos grupos de investigación. Cada vez que era necesaria una consulta comparando localizaciones o estaciones, los datos eran procesados a mano. Con esto se quiere decir que una persona leía los archivos de texto que generaba el aparato y los transformaba en Excel para su posterior tratamiento. El tamaño tan grande de datos dificultaba el análisis de grandes temporadas de datos, perdiendo el valor añadido que supone disponer de tan amplia base de datos.

Por eso se ha decidido en este trabajo afrontar el siguiente objetivo:

- Crear un programa en java que lea archivos .txt y procese los datos de manera coherente para su posterior utilización.

- Conectar el programa Java con una base de datos para poder almacenar los datos procesados.

- Visualizar de forma gráfica los datos contenidos en la base de datos mediante una aplicación web (Fig 1).

- Crear un login; solamente los usuarios autorizados tendrán acceso a la visualización de los datos.

- Crear un programa muy sencillo para que incluso un usuario inexperto pueda utilizarlo.

Objetivos secundarios.

- Ayudar a que el usuario pueda visualizar los datos de forma que le resulten más fáciles la interpretación.

- Crear un acceso seguro al programa para evitar la vulnerabilidad de los datos.

- Crear una guía de utilización del programa para facilitar el trabajo a usuarios inexpertos.

- Implantar el programa en el servidor de la universidad para lograr un acceso, extendido.

- Establecer un canal de ayuda de cara al usuario final para la solución de problemas inesperados.

- Crear una aplicación estable y segura.

\section{Material y métodos}

El proyecto se ha realizado dividiendo el trabajo en 3 fases:

1. Primera fase: Un integrante del grupo desarrolla una aplicación en Java que tiene como función principal leer los informes generados por el disdrómetro (Java, 2017). Después de leer estos datos, tenemos que procesarlos y para ello usamos un algoritmo que nos permite saber la velocidad y el diámetro de las precipitaciones que es lo que más nos interesa en nuestro proyecto. Una vez que hemos obtenido toda la información necesaria procedemos a conectar la aplicación con una base de datos MySQL.

2. Segunda fase: En esta segunda fase un segundo integrante del grupo crea la base de datos MySQL de forma local para simplificar la conexión y luego poder subirla a la 
web. Las tablas siguen la misma estructura que los datos recogidos por el disdrómetro para facilitar la comprensión de los datos.

3. Tercera fase: En esta tercera y última fase el tercer integrante del grupo procede a crear una aplicación web que utilizaremos para mostrar los resultados de forma gráfica. Esta aplicación se conecta con la base de datos anteriormente mencionada usando PHP y sentencias SQL. Como lo que más nos interesa es poder estudiar las precipitaciones en un periodo de tiempo, decidimos mostrar todos estos datos de forma clara usando la librería Highcharts, que nos mostrará gráficamente todos estos datos en porciones de tiempo (Guo, F.; Liang, Y. 2016.).

4. Por último, introducimos en la aplicación web nuestro programa de lectura y transformación de los datos para que cuando se necesite introducir nuevos registros en la base de datos se haga siguiendo el mismo patrón utilizado anteriormente.

En la parte central de nuestra página principal se encuentra una serie de tres imágenes que nos indican, mediantes los dibujos que hay en ellas, la información que contienen sobre las tablas y gráficas que se presentan. Debajo de estas imágenes existen unos links en azul que nos llevarán a la página donde se encuentran las gráficas que el propio link y el título de las imágenes nos indica.

Debido a que el almacenamiento de una cantidad de datos muy grande puede ocasionar numerosos problemas, además de un tiempo de espera de las consultas muy largo, se ha procedido a dividir la consulta de información separando los datos y clasificándolos por años, meses y días.

Los datos de nuestro trabajo están guardados en una base de datos con un servidor local. El diseño de la plataforma en forma modular permite la implementación en el futuro de botones automáticos para el añadido de distintos cálculos automáticos con los datos. Además, se puede mejorar nuestra aplicación a la hora de optimizar nuestro código, mejorando la eficiencia en los bucles y consultas a la base de datos. Se está trabajando en mejorar la complejidad de los algoritmos para reducir el tiempo de consulta a la base de datos.

A la hora de crear una base de datos almacenados para su posterior uso, surgieron la mayoría de los problemas: en primer lugar, al decidir el tipo de formato que debía utilizarse en los registros de la base de datos. Al leer y procesar los datos, a veces había diferencias entre las fechas que se indicaban en las cabeceras de los archivos, asignados por el ordenador, y las que asignaba el propio disdrómetro, por lo que al intentar subir estos datos, no coincidían los formatos de origen y de destino y se producían errores, que fueron subsanados. Más adelante, las dificultades procedían del enorme tamaño de los datos almacenados, que eran más de 14 millones y medio de registros y pesaban un total de $883 \mathrm{MB}$, por lo que requerían tiempos de consulta muy elevados, que hacían retrasar la ejecución del programa. Cuando mostrábamos datos en rangos de un par de años la aplicación tardaba 4 o 5 min en responder, por lo que los tiempos de carga no eran eficientes. La solución fue indexar la base de datos por el campo fecha y de esta forma redujimos los tiempos de carga.

Una vez solucionado este aspecto, la visualización de los datos almacenados en gráficas fue relativamente sencilla. El programa permite en la página principal visionar toda 
la información y una serie de gráficas donde consultar los datos obtenidos por el disdrómetro (Fig 1), y en una opción posterior poder seleccionar el visionado de periodos de tiempo concretos (Fig 2a).

\section{Resultados}

Un usuario no experto podrá consultar los datos sin ningún problema y de forma sencilla (Fig 2b). La aplicación web tiene un entorno sencillo y agradable, accesible para cualquier usuario.

La protección de los datos mediante un login para regular la entrada a la aplicación a personas autorizadas fue sencilla, y además se insertó código SQL para que ninguna persona pudiera modificar la base de datos.

El objetivo último será recargar la plataforma con los nuevos datos recogidos para que esta base de datos alojada en el servidor web de la Universidad pueda actualizarse y comprender los fenómenos y características de los eventos de lluvia en la ciudad de León.
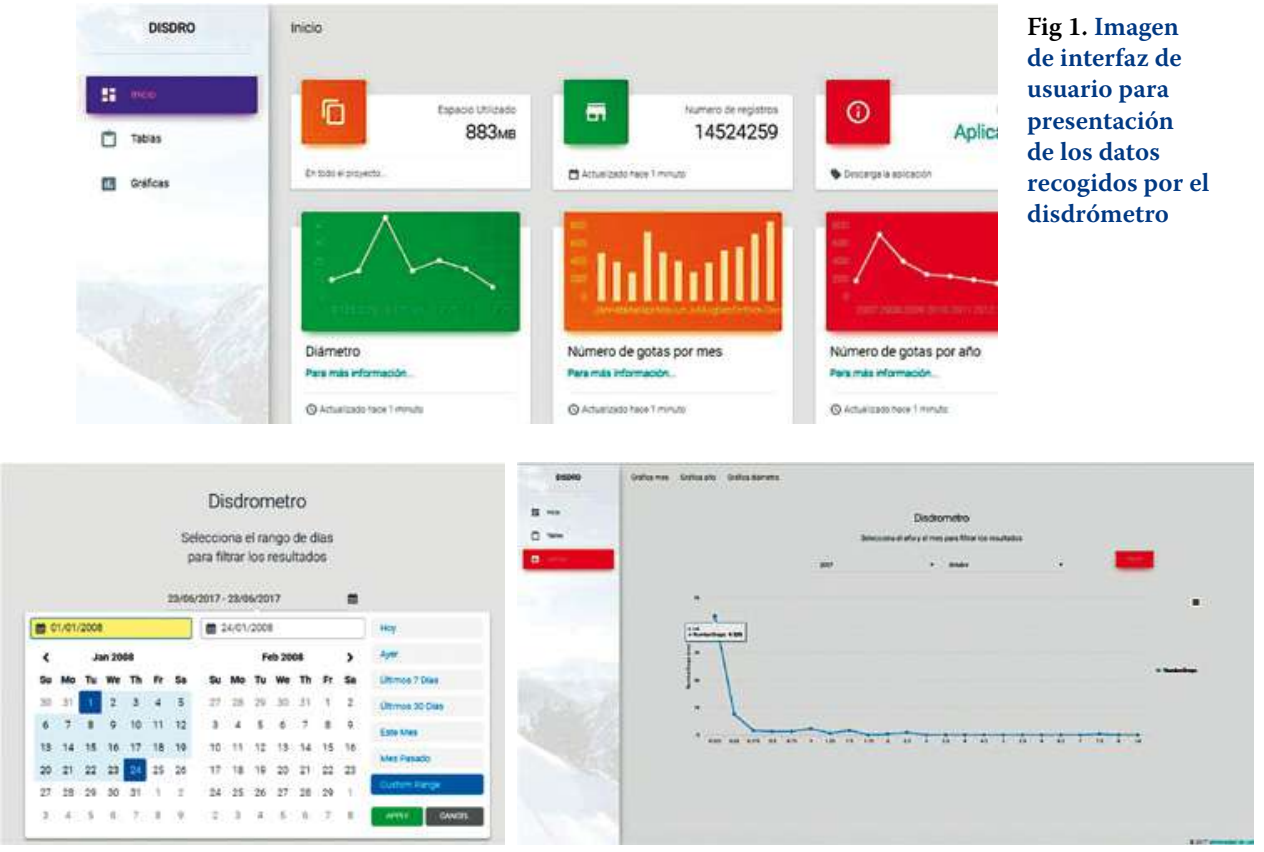

Fig 2. Interfaz de la aplicación para a) la extracción y análisis de datos por periodos concretos y b) análisis de distribuciones de tamaños de gotas en periodos de tiempo concretos. 


\section{Conclusiones}

El desarrollo de esta herramienta ha permitido crear un programa en Java que lee archivos .txt y procesa los datos de manera coherente para su posterior utilización.

Este programa es capaz de almacenar grandes cantidades de datos y ordenarlos cronológicamente. Además permite hacerle consultas y se conecta mediante el programa Java con una base de datos para poder almacenar los datos procesados. Como resultados ofrece tablas de datos elaborados y gráficos realizados mediante una aplicación web. Este instrumento además consigue proteger la propiedad de los datos mediante clave.

\section{Agradecimientos}

Este estudio ha sido parcialmente financiado por el Ministerio Español de Ciencia e Innovación (Proyecto TEC2014-57821-R y CGL2014-52556-R). María Fernández-Raga agradece además la beca postdoctoral José Castillejo del Ministerio Español de Ciencia e Innovación (Beca CAS16/00385).

\section{Referencias}

- Chen, B., Wang, J., Gong, D., 2015: Raindrop Size Distribution in a Midlatitude Continental Squall Line Measured by Thies Optical Disdrometers over East China Journal Of Applied Meteorology And Climatology 55; 3, 621-634.

- Frasson, R.P.M., da Cunha, L.K.; Krajewski, W.F. 2014: Assessment of the Thies optical disdrometer performance Atmospheric Research 101 (1-2) : 237-255 DOI: 10.1016/j.atmosres.2011.02.014.

- Guo, F.; Liang, Y. 2016. Design and Implementation of the Network Video Data Acquisition System. Proceedings Of 2016 Ieee 7th International Conference On Software Engineering And Service Science (Icsess 2016) International Conference on Software Engineering and Service Science, 513-516.

- Java, 2017 «Capítulo 1. Introducción (Introducción a JavaScript)». [En línea]. Disponible en: http://librosweb.es/libro/javascript/capitulo_1.html. [Accedido: 27-jun-2017]. 